\title{
The utility of selected questionnaires in the assessment of fatigue, depression and health quality in post-sarcoidosis fatigue syndrome
}

The authors declare no financial disclosure

\begin{abstract}
Introduction: The nature of post-sarcoidosis fatigue syndrome (PSFS) is unknown and tools for the assessment of health quality $(\mathrm{HO})$ in these patients have not been fully assessed. The aim was to validate the Polish version of sarcoidosis health questionnaire (SHO) and verify the association of $\mathrm{HO}$ with fatigue and depressive symptoms among Polish patients with PSFS.

Material and methods: 71 patients with sarcoidosis (34 women, the mean age 47) were divided to: PSFS ( $n=21$ ), active sarcoidosis ( $\mathrm{S}-\mathrm{A}, \mathrm{n}=27$ ) and sarcoidosis with complete remission ( $\mathrm{S}-\mathrm{R}, \mathrm{n}=23$ ) groups. Fatigue Assessment Scale (FAS) was used to define significant fatigue ( $\geq 22$ points). Polish version of $S H Q$ was prepared by the authors and validated. Beck Depression Index (BDI) and Patient Health Questionnaire 9 (PHQ-9) were used to evaluate self-reported depressive symptoms.

Results: Polish version of $\mathrm{SHO}$ was proved reliable and valid. $\mathrm{HO}$ was worse and depressive symptoms were more frequent in PSFS and S-A when compared with S-R group. SHO total score correlated negatively with depressive symptoms $(r=-0.787$ for $\mathrm{BDI}$ and $r=-0,755$ for PHQ-9, $p<0.01)$. A negative correlation between $\mathrm{SHO}$ and FAS score was found $(r=-0.784, p<0.01)$. FAS score correlated with depressive symptoms ( $r=0.726$ for BDI and $r=0.755$ for PHQ-9, $p<0.01$ ).

Conclusion: Polish version of $\mathrm{SHO}$ is a valuable tool for the assessment of $\mathrm{HO}$ in sarcoidosis. $\mathrm{HO}$ is impaired in PSFS comparing to patients with complete remission, but is comparable to active sarcoidosis. Depressive symptoms impact $\mathrm{HO}$ and may influence perception of fatigue. Both fatigue and depression have a negative impact on $\mathrm{HO}$ in sarcoidosis.
\end{abstract}

Key words: post-sarcoidosis fatigue syndrome, sarcoidosis, health quality, fatigue, FAS

Adv. Respir. Med. 2017; 85: 313-321

\section{Introduction}

Sarcoidosis is a chronic inflammatory disease of unknown etiology, which mainly affects intrathoracic lymph nodes and lung parenchyma, with possible involvement of other organs. Noncaseating granulomas consisting of epithelioid and giant cells are found in histopathological examination of tissue samples taken from compromised organs $[1,2]$. The overall prognosis is good, as about $60 \%$ of patients, regardless clinical phenotype, experience spontaneous remissions.
Deaths - mainly due to lung fibrosis, heart involvement or neurosarcoidosis - are rare [1, 2]. The clinical presentation is variable. Symptomless course with accidental diagnosis is frequent. Many patients present symptoms resulting from specific organ involvement or from other disease-related pathological processes, like impairment of calcium metabolism. Many general symptoms are non-specific, for example weight loss, elevated temperature, sweats, myalgia or arthralgia [1-3]. Fatigue is one of the most frequently reported symptoms in active sarcoidosis $(50-70 \%$ of pa-

Address for correspondence: Wojciech J. Piotrowski, Department of Pneumology and Allergy, $1^{\text {st }}$ Chair of Internal Medicine, Medical University of Lodz, Poland, e-mail: wojciech.piotrowski@umed.lodz.pl

DOI: 10.5603/ARM.2017.0054

Received: 23.10.2017

Copyright (C) 2017 PTChP

ISSN 2451-4934 
tients according to different sources), and is frequently associated with other general symptoms, for instance myalgia, arthralgia, night sweats [2-4]. It severely impacts quality of life [4] and is difficult to treat $[5,6]$.

Patients with diversity of acute and chronic diseases frequently report fatigue. Chronic fatigue is reported by patients infected with Ebstein-Bar (EBV), human immunodeficiency (HIV), hepatitis C (HCV) viruses, patients with neoplastic disease, rheumatoid arthritis and systemic sclerosis, multiple sclerosis, fibromyalgia, obstructive sleep apnea and many others $[7,8]$. American Centers for Disease Control and Prevention (CDC) proposed a term chronic fatigue syndrome (CFS), defined as a "debilitating and complex disorder characterized by intense fatigue that is not improved by bed rest and that may be worsened by physical activity or mental exertion" [7].

In view of the above, chronic fatigue in the course of active sarcoidosis is not an unexpected symptom. However, it is especially intriguing, that it may appear and persist for a long period of time in patients with radiological and clinical remission. Sharma used a term post-sarcoidosis fatigue syndrome (PSFS) and estimated its frequency to about $5 \%$ [9]. The origin of fatigue in sarcoidosis is still unknown. In active disease several factors should be considered, for instance systemic inflammation, muscle involvement, extrapulmonary locations, comorbidities, pulmonary hypertension, impaired lung function, steroids and other drugs used for the treatment of sarcoidosis, and psychological factors [10]. The pathogenesis of PSFS is even more mysterious [11, 12]. Depression and anxiety is frequent in patients with active sarcoidosis [12-14]. There are few reports pointing out the possible link between fatigue with depression. Goracci et al. [15] reported on strong relationship between sarcoidosis and its negative impact on quality of life. De Klejn et al. [16] found, that fatigue is related to depression in $34-36 \%$ and to anxiety in $43-46 \%$ of patients suffering from active sarcoidosis. The problem of underlying depression and its influence on fatigue has not been, however, studied in patients with sarcoidosis remission. In addition to that, fatigue is difficult to quantify objectively, and different measures have been applied for its assessment so far. Fatigue Assessment Scale is a self-administered questionnaire quantified and validated in the assessment of fatigue in different diseases, including sarcoidosis [17].

Assessment of health-related quality of life may be as important as assessment of depressive symptoms and fatigue among patients suffering from sarcoidosis [15]. Sarcoidosis Health Questionnaire (SHQ) is one of the most popular psychometric tools assessing quality of life designed for patients with sarcoidosis [18]. The SHQ questionnaire, as well as other questionnaires used for the assessment of accompanying fatigue and depression have not been used in patients with PSFS. Also, no validation of Polish version of SHQ has been published up to date.

Therefore, the aim of this study was to validate the Polish version of SHQ and verify the association of quality of life with fatigue and depressive symptoms among patients with PSFS.

\section{Material and methods}

\section{Participants}

The study included 71 patients with sarcoidosis (34 women and 37 men) aged $28-80$ years (the mean age 46,82 years), who reported to Outpatient Clinic and to Department of Pneumology and Allergy of the First Clinical University Hospital in Lodz. Sarcoidosis was diagnosed in accordance with the criteria of the American Thoracic Society and World Association for Sarcoidosis and Other Granulomatous Disorders (ATS/WASOG) [19]. The study group was divided in three cohorts:

1) Group A, consisting of 21 patients with clinical and radiological remission and symptoms of fatigue, representing post-sarcoidosis fatigue syndrome (PSFS) group. These patients had normal chest x-ray, showing complete remission of previous radiological changes. In doubtful cases chest high resolution computed tomography (HRCT) was performed to confirm complete radiological resolution. None of the patients from this group had signs of extrapulmonary organs involvement or abnormal laboratory tests results. Fatigue assessment scale (FAS) was used to define patients with significant fatigue, and the score of $\geq 22$ was used as a threshold [17].

2) Group B, consisting of 23 patients with clinical remission without fatigue, representing a group of complete remission (SR). These patients had normal chest $\mathrm{x}$-ray, showing complete remission of previous radiological changes, had no signs of extrapulmonary organs involvement, normal laboratory tests results, and no symptoms. The lack of significant fatigue symptoms was defined by the FAS score $\leq 21$ [17];

3) Group C, consisting of 27 patients with active sarcoidosis (AS). For the purpose of this study the disease was considered active, when the 
patient presented with new-onset radiological changes or radiological progression, reported acute symptoms (fever, arthritis, erythema nodosum), or respiratory symptoms (cough, dyspnea, chest tightness) in the presence of typical radiological picture, when other causes of these symptoms were excluded. In patients with BAL performed in the previous 3 months, increased \% of lymphocytes above $20 \%$ was considered abnormal, and spoke for activity. 11 patients from this group presented symptoms of Löfgren syndrome.

None of the patients smoked cigarettes. None of the patients in the study had been treated with oral corticosteroids or immunosuppressive drugs. No symptoms of chronic concomitant diseases which could provoke and increase fatigue or contribute to patient mental health were reported. Also no symptoms of acute infection were observed in the patients during and prior to the study. None of the women participating in the study was pregnant at the time of the study. There were no patients with extrapulmonary sarcoidosis. We followed the recruitment protocol according to which, patients with radiological and clinical remission were screened for chronic fatigue with FAS questionnaire first, and after completing the desired number of patients in each group (S-R and PSFS), the third group of patients with active sarcoidosis was selected from patients consecutively presenting at the centre, with regard of inclusion/ /exclusion criteria written above. Estimation of the frequency of chronic fatigue or PSFS in the entire population of sarcoidosis patients was not the aim of our study.

In all the participants spirometry (Lungtest 1000, MES, Poland) and diffusing capacity for carbon monoxide study (DLCo) (MES, Poland) were performed in accordance with the ATS/ERS and Polish guidelines [20, 21]. The obtained results of forced vital capacity (FVC), forced expiratory volume in 1 second $\left(\mathrm{FEV}_{1}\right)$, and DLco (corrected for hemoglobin concentration - DLCOc) were expressed as a percentage of predicted values.

Chest $\mathrm{x}$-ray was performed in all patients. In patients with active sarcoidosis the Scadding classification [1] was used to define radiological stage (stage 1 - enlarged hilar lymph nodes; stage 2 - enlarged hilar lymph nodes with parenchymal changes; stage 3 - parenchymal changes without enlarged hilar lymph nodes).

\section{Questionnaires}

The Polish version of the Sarcoidosis Health Questionnaire was devised by means of a standard psychometric procedure, i.e. the forward-backward translation [22]. Two pulmonologists translated the SHQ independently and consulted on the final content, which was counter-translated and compared with the original SHQ. The questionnaire comprises 29 items divided into three domain: Daily Functioning (DF, 13 items), Physical Functioning (PF, 6 items) and Emotional Functioning (EF, 10 items). Each item is scored on a 7-point Likert scale. Total score and scores in each domain are sums of the scores divided by the number of items. Higher scores in SHQ reflect better health-related quality of life among patients with sarcoidosis.

The Beck Depression Index (BDI) was used to evaluate self-reported depressive symptoms $[23,24]$. The Polish adaptation was developed by Zawadzki et al. [25]. BDI consists of 21 items referring to criteria of depression, e.g. anhedonia, lack of energy or diminished mood. Each item has four possible items, scored from 0 to 3 . Psychiatric consultations were not performed.

The Patient Health Questionnaire 9 (PHQ-9) is another self-administered measure of depressive symptoms. It was developed for the purposes of brief screening for mood disorders in clinical practice by Kroenke et al. [26]. Polish translation of PHQ-9 is available online [27]. The questionnaire consists of nine items, scored from 0 to 3 , depending on the reported frequency of the symptoms. Higher scores indicate greater severity of the self-reported depressive symptoms in both BDI and PHQ-9.

The (C) FAS (Fatigue Assessment Scale: ILD care foundation (www.ildcare.nl) was used to assess the severity of fatigue in the studied sample. It was developed by de Vries et al. [17] and the Polish version (prepared by A. Dubaniewicz) is available online [28]. It comprises ten items divided into two five-item subscales: Physical Fatigue (FAS-P) and Mental Fatigue (FAS-M). Higher scores suggest greater severity of the experienced fatigue.

\section{Statistical analysis}

Continuous variables were characterized by mean value $(\mathrm{M})$ with respective standard deviation (SD). Cronbach's alpha for the SHQ were calculated to verify the reliability of the Polish version of the questionnaire. The validity of the SHQ was assessed by means of inter-group comparisons (active sarcoidosis vs PSFS vs control) and correlations with scores of other psychometric tools and the lung function test results.

Analysis of variance was used for the inter-group comparisons. The homogeneity of vari- 
Table 1. Group characteristics and comparison of variables of interest between studied groups of patients with active sarcoidosis (AS), post-sarcoidosis fatigue syndrome (PSFS) and patients with sarcoidosis in remission without fatigue (S-R)

\begin{tabular}{|c|c|c|c|c|c|}
\hline & Total $(n=71)$ & $S-R(n=23)$ & PSFS (n = 22) & $S-A(n=26)$ & p \\
\hline Female (\%) & 34 (47.9\%) & $14(60.9 \%)$ & $7(28.3 \%)$ & $14(53.8 \%)$ & 0.059 \\
\hline Age & $46.82 \pm 12.3$ & $43.78 \pm 12.9$ & $45.05 \pm 12.44$ & $51 \pm 10.86$ & 0.087 \\
\hline $\mathrm{FEV}_{1}$ & $88.83 \pm 11.48$ & $96.22 \pm 8.62$ & $86.95 \pm 8.94^{\dagger}$ & $83.88 \pm 12.56^{\dagger}$ & $<0.001$ \\
\hline FVC & $91.92 \pm 12.56$ & $98.57 \pm 12.86$ & $91.05 \pm 9.56$ & $86.77 \pm 12.27^{\dagger}$ & 0.003 \\
\hline $\mathrm{FEV}_{1} / \mathrm{FVC}$ & $0.77 \pm 0.05$ & $0.79 \pm 0.04$ & $0.77 \pm 0.05$ & $0.76 \pm 0.06$ & 0.077 \\
\hline DLCO & $93.58 \pm 12.35$ & $96.87 \pm 9.11$ & $93.14 \pm 9.95$ & $91.04 \pm 15.94$ & 0.255 \\
\hline $\begin{array}{l}\text { Radiological stage* } \\
1 / 2 / 3 / 4\end{array}$ & $30 / 37 / 4 / 0$ & $10 / 12 / 0 / 0$ & $16 / 7 / 0 / 0$ & $4 / 18 / 4 / 0$ & \\
\hline SHQ-PF & $4.78 \pm 1.1$ & $5.78 \pm 0.57$ & $4.24 \pm 1.04^{*}$ & $4.34 \pm 0.9^{*}$ & $<0.001$ \\
\hline SHO-EF & $4.76 \pm 0.99$ & $5.86 \pm 0.53$ & $4.16 \pm 0.59^{*}$ & $4.29 \pm 0.74^{*}$ & $<0.001$ \\
\hline SHQ-DF & $4.57 \pm 1.07$ & $5.75 \pm 0.41$ & $3.88 \pm 0.75^{*}$ & $4.11 \pm 0.81^{*}$ & $<0.001$ \\
\hline SHO total & $4.68 \pm 0.97$ & $5.79 \pm 0.41$ & $4.05 \pm 0.57^{*}$ & $4.22 \pm 0.73^{*}$ & $<0.001$ \\
\hline $\mathrm{BDI}$ & $8.76 \pm 6.07$ & $3.74 \pm 1.6$ & $12.18 \pm 6.93^{*}$ & $10.31 \pm 4.95^{*}$ & $<0.001$ \\
\hline PHO-9 & $5.37 \pm 4.04$ & $1.83 \pm 1.23$ & $7.95 \pm 4.75^{*}$ & $6.31 \pm 2.68^{*}$ & $<0.001$ \\
\hline FAS-P & $12.17 \pm 4.21$ & $7.78 \pm 1.35$ & $15.82 \pm 3.45^{* \neq}$ & $12.96 \pm 2.81^{*}$ & $<0.001$ \\
\hline FAS-M & $9.97 \pm 2.96$ & $7.09 \pm 1.2$ & $11.86 \pm 2.83^{* \ddagger}$ & $10.92 \pm 2.19^{*}$ & $<0.001$ \\
\hline FAS total & $22.13 \pm 6.76$ & $14.83 \pm 1.97$ & $27.68 \pm 5.45^{* \ddagger}$ & $23.88 \pm 4.49^{*}$ & $<0.001$ \\
\hline
\end{tabular}

Presented as mean values with standard deviations, with an exception for sex. SHO — Sarcoidosis Health Questionnaire; PF — Physical Functioning; EF — Emotiona Functioning; DF — Daily Functioning; BDI — Beck Depression Inventory; PHQ-9 — Patient Health Questionnaire; FAS — Fatigue Assessment Scale; FAS-P — Physical Fatigue; FAS-M - Mental Fatigue; FEV 1 — forced expiratory volume in 1 second; FVC — forced vital capacity; DLCO — diffusing capacity or of the lung for carbon monoxide; $p$ - probability in the test of significance; ${ }^{*} p<0.001$ vs $S-R ; t p<0.01$ vs $S-R ;{ }^{\ddagger} p<0.01$ vs $S-A$

ance was checked with Levene's test. The F test with post-hoc Tukey test were used if the variances between groups were assumed homogeneous. If the variance was assumed non-homogeneous, Welch test with post-hoc Dunnett T3 test was used. The association between two continuous variables was assessed with Pearson correlation quotients. The link between two continuous variables was assessed with Spearman correlation quotients in the subgroup analysis due to non-normal distribution of BDI and PHQ-9 (verified with Shapiro-Wilk test). The correlation coefficients were compared with an online tool [29]. Results were considered statistically significant when $p<0.05$. SPSS 24.0 (IBM, USA and Predictive Solutions, Poland) was used for all analyses.

\section{Results}

Clinical characteristics of study and control groups is presented in Table 1 . The groups were not different it terms of age and gender. Patients with PSFS and active sarcoidosis had significantly lower $\mathrm{FEV}_{1} \%$ predicted comparing to patients with complete remission and FVC\% predicted was lower in active sarcoidosis comparing to PSFS group and complete remission group.

\section{Reliability of Polish adaptation of SHQ}

Cronbach's alpha for the SHQ was 0.95. Coefficient's values for the DF, PF and EF domains were $0.91,0.78$ and 0.87 , respectively.

\section{Validity of Polish adaptation of SHQ}

Mean scores on PF, DF and EF scales and mean SHQ total score were higher among S-R patients than in PSFS patients and S-A patients (Fig. 1). Detailed results are shown in Table 1. Results of FAS, BDI and PHQ in all 3 groups of patients is shown in Figures 2-4.

There was a moderate, negative and statistically significant correlation between SHQ-PF and age ( $\mathrm{r}$ $=-0.308$ ). SHQ-PF, EF, DF and total score correlated strongly, negatively and significantly with depressive symptoms indicators, i.e. BDI $(\mathrm{r}=-0.660$ to $\mathrm{r}$ $=-0.804, p<0.01)$ and PHQ-9 $(r=-0.676$ to $r=$ $-0,755, p<0.01)$. A strong, negative and statistically significant correlations were also found between SHQ-PF, EF, DF and total score and chronic fatigue indicators, i.e. FAS total score $(r=-0.711$ to $\mathrm{r}=$ 


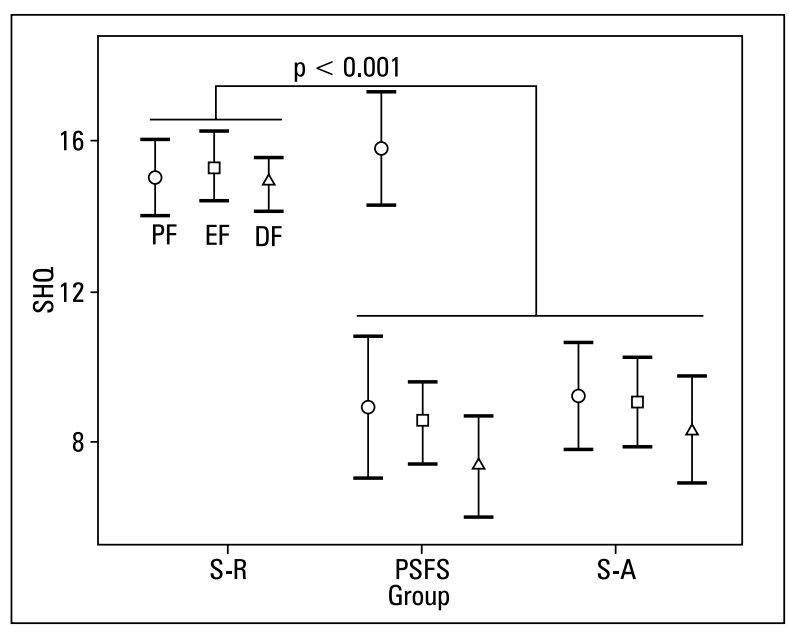

Figure 1. Mean scores on Sarcoidosis Health Questionnaire (SHO) scales compared between the studied groups of patients with active sarcoidosis (AS), post-sarcoidosis fatigue syndrome (PSFS) and patients with sarcoidosis in remission without fatigue (S-R). PF — Physical Functioning (circle); EF — Emotional Functioning (square); DF — Daily Functioning (triangle). The bars represent the $95 \%$ Confidence Intervals for the mean values

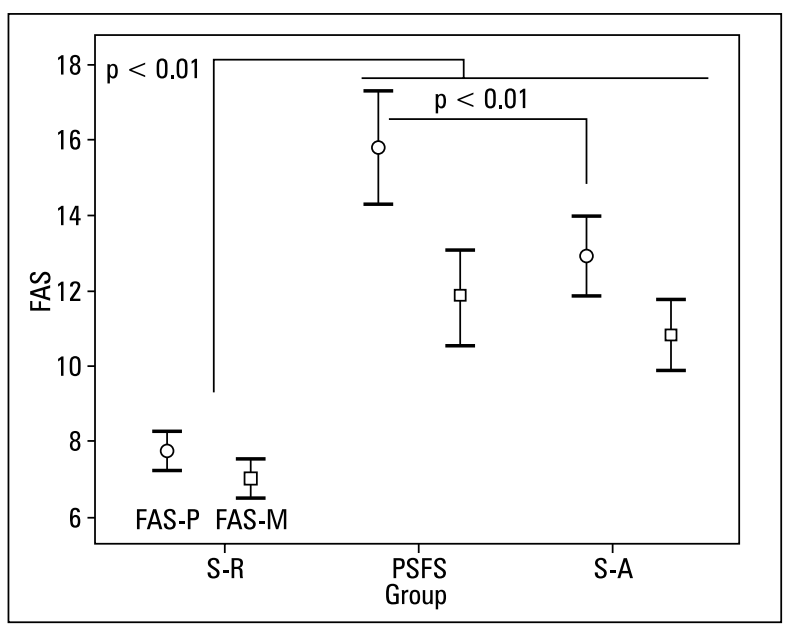

Figure 2. Mean scores on Fatigue Assessment Scale (FAS) scales compared between the studied groups of patients with active sarcoidosis (AS), post-sarcoidosis fatigue syndrome (PSFS) and patients with sarcoidosis in remission without fatigue (S-R). FAS-P — Physical Fatigue (circle); FAS-M — Mental Fatigue (square). The bars represent the $95 \%$ Confidence Intervals for the mean values

-0.784, $\mathrm{p}<0.01)$ and its subscales, namely PA $(\mathrm{r}=$ -0.770 to $\mathrm{r}=-0.832, \mathrm{p}<0.01)$ and MA $(\mathrm{r}=-0.702$ to $r=-0.779, p<0.01)$. No statistically significant correlations were found between SHQ scores and lung function indicators $\left(\mathrm{FEV}_{1}, \mathrm{FVC}, \mathrm{FEV}_{1} / \mathrm{FVC}\right.$ or $\mathrm{DL}_{\mathrm{CO}}$ ). Table 2 shows detailed results.

\section{Correlates of the FAS score}

Apart from the aforementioned correlations with SHQ scores, FAS total score correlated significantly and positively with age, BDI score and

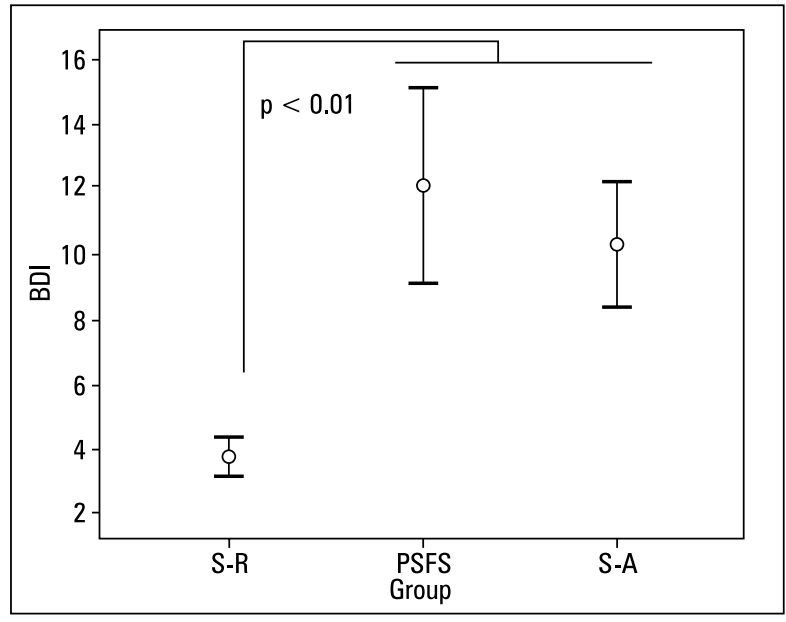

Figure 3. Mean scores on Beck Depression Inventory (BDI) compared between the studied groups of patients with active sarcoidosis (AS), post-sarcoidosis fatigue syndrome (PSFS) and patients with sarcoidosis in remission without fatigue (S-R). The bars represent the $95 \%$ Confidence Intervals for the mean values

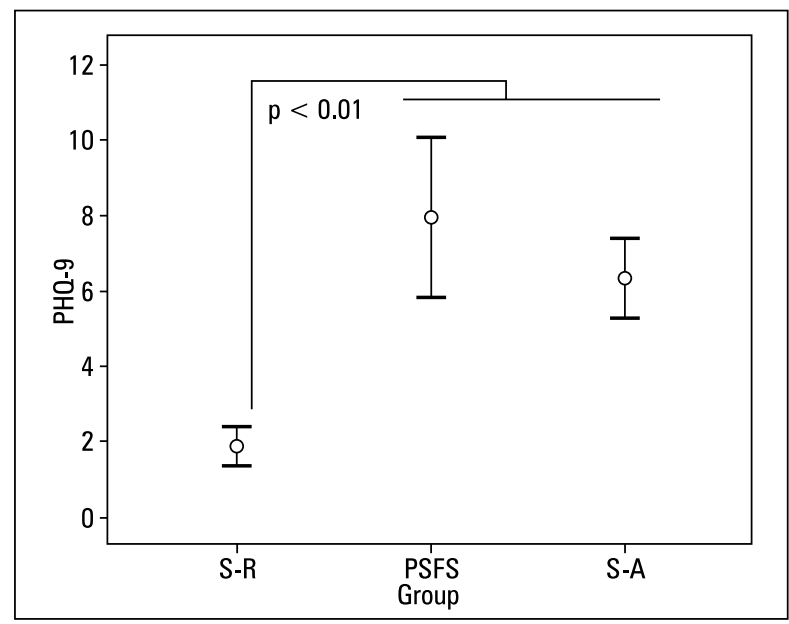

Figure 4. Mean scores on Patient Health Questionnaire (PHQ-9) compared between the studied groups of patients with active sarcoidosis (AS), post-sarcoidosis fatigue syndrome (PSFS) and patients with sarcoidosis in remission without fatigue (S-R). The bars represent the 95\% Confidence Intervals for the mean values

PHQ-9 score. FAS Physical Fatigue scale score correlated with BDI score and PHQ-9, while FAS Mental Fatigue scale score was positively associated with age and both BDI and PHQ-9 scores. Also, there was a weak, yet statistically significant and negative correlation between FAS-M score and $\mathrm{FEV}_{1}$. No other statistically significant correlation was found between FAS scores and chosen lung function parameters $\left(\mathrm{FEV}_{1}, \mathrm{FVC}, \mathrm{FEV}_{1} / \mathrm{FVC}\right.$ or $\mathrm{DL}_{\mathrm{CO}}$ ). Table 3 contains detailed results on the described correlation quotients. 
Table 2. Pearson correlation coefficients of Sarcoidosis Health Questionnaire total score and its dimensions scores with age and chosen indicators of depressive symptoms, chronic fatigue and lung capacity

\begin{tabular}{lcccc}
\hline & SHO-PF & SHQ-EF & SHO-DF & SHO total \\
\hline Age & $-0.308^{*}$ & -0.146 & -0.157 & -0.200 \\
BDI & $-0.660^{*}$ & $-0.804^{*}$ & $-0.715^{*}$ & $-0.787^{*}$ \\
PHQ-9 & $-0.690^{*}$ & $-0.748^{*}$ & $-0.676^{*}$ & $-0.755^{*}$ \\
FAS-P & $-0.778^{*}$ & $-0.775^{*}$ & $-0.770^{*}$ & $-0.832^{*}$ \\
FAS-M & $-0.743^{*}$ & $-0.702^{*}$ & $-0.732^{*}$ & $-0.779^{*}$ \\
FAS total & $-0.717^{*}$ & $-0.764^{*}$ & $-0.711^{*}$ & $-0.784^{*}$ \\
FEV 1 & 0.150 & 0.194 & 0.155 & 0.180 \\
FVC & 0.209 & 0.151 & 0.104 & 0.153 \\
FEV $_{1} /$ FVC & 0.075 & 0.103 & 0.065 & 0.085 \\
LL $_{\text {c0 }}$ & 0.089 & 0.091 & 0.111 & 0.107 \\
\hline
\end{tabular}

PF — Physical Functioning; EF — Emotional Functioning; DF — Daily Functioning; BDI — Beck Depression Inventory; PHQ-9 — Patient Health Questionnaire; FAS — Fatigue Assessment Scale; FAS-P — Physical Fatigue; FAS-M — Mental Fatigue; FEV 1 — forced expiratory volume in 1 second; FVC — forced vital capacity; DLCO — diffusing capacity of the lung for carbon monoxide; ${ }^{*} \mathrm{p}<0.01$ (two-tailed)

Table 3. Pearson correlation coefficients of Fatigue Assessment Scale total score and its dimensions scores with age and chosen indicators of depressive symptoms and lung capacity

\begin{tabular}{lccc}
\hline & FAS total & FAS-P & FAS-M \\
\hline Wiek & $0.247^{*}$ & 0.212 & $0.266^{*}$ \\
BDI & $0.726^{* *}$ & $0.670^{* *}$ & $0.702^{* *}$ \\
PHQ-9 & $0.788^{* *}$ & $0.768^{* *}$ & $0.705^{* *}$ \\
FEV 1 & -0.221 & -0.190 & $-0.235^{*}$ \\
FVC & -0.195 & -0.184 & -0.182 \\
FEV $/$ FVC & -0.168 & -0.111 & -0.226 \\
DLCO & -0.083 & -0.056 & -0.103 \\
\hline
\end{tabular}

BDI — Beck Depression Inventory; PHQ-9 — Patient Health Questionnaire; FAS — Fatigue Assessment Scale; FAS-P — Physical Fatigue; FAS-M — Mental Fatigue; $\mathrm{FEV}_{1}$ - forced expiratory volume in 1 second; FVC — forced vital capacity; DLCO - diffusing capacity or of the lung for carbon monoxide; * $\mathrm{p}<$ 0.05 (two-tailed); ${ }^{* *} \mathrm{p}<0.01$ (two-tailed)

\section{Subgroup analysis - FAS score vs depressive symptoms indicators}

In the PSFS subgroup, there were positive and statistically significant correlations between the FAS dimensions and both BDI and PHQ-9 scores $(\mathrm{r}=0.448$ to $0.693, \mathrm{p}<0.05)$, with an exception for the correlation between BDI and FAS-M $(r=0.407, p=0.06)$. Similarly, in the S-A subgroup, FAS-M, FAS-P and FAS total score correlated positively and statistically significantly with BDI and PHQ-9 ( $\mathrm{r}=0.456$ to $0.748, \mathrm{p}<$ 0.05). There was an exception - the correlation between FAS-P and BDI was not found to be statistically significant $(\mathrm{r}=0.356, \mathrm{p}=0.07)$.
Despite no statistically significant difference of the aforementioned correlation quotients between the PSFS and control subgroups, three trends may be pointed out: the correlation quotient between PHQ-9 and FAS-P is higher in the PSFS group than in the $S$-A group $(\mathrm{r}=0.673$ vs $\mathrm{r}=0.456$, respectively, $\mathrm{p}=0.30$ ).

FAS-M correlates with BDI and PHQ-9 weaker in the PSFS group than in the S-A group (BDI: $r$ $=0.407$ vs $0.658, p=0.25$; PHQ-9: $r=0.464$ vs $0.749, \mathrm{p}=0.13$ ).

The correlations between FAS dimensions and depressive symptoms indicators in the remitted sarcoidosis group were found weak and statistically insignificant. Thus, it was assumed that the associations between those variables is either weaker than in the PSFS and active sarcoidosis control subgroup or absent (Table 4).

\section{Discussion}

This study was designed to validate Polish version of SHQ and to assess the relationship between health quality, depressive symptoms and fatigue. We also studied the level of health quality impairment and the intensity of depressive symptoms in patients with persistent chronic fatigue regardless clinical and radiological evidence of remission (post-sarcoidosis fatigue) and compared it to control groups of patients with complete remission and active disease. Fatigue syndrome was defined by high total score of FAS equal to or higher than 22 points. In our study we positively validated the Polish version of SHQ. We also fo- 
Table 4. Comparison of Spearman correlation coefficients of Fatigue Assessment scale total score and its dimensions scores with the chosen indicators of depressive symptoms between studied groups of patients with active sarcoidosis (AS), post-sarcoidosis fatigue syndrome (PSFS) and patients with sarcoidosis in remission without fatigue (S-R)

\begin{tabular}{|c|c|c|c|c|c|c|}
\hline & \multicolumn{2}{|c|}{$S-R(n=23)$} & \multicolumn{2}{|c|}{ PSFS (n = 22) } & \multicolumn{2}{|c|}{$S-A(n=26)$} \\
\hline & BDI & PHO-9 & BDI & PHO-9 & BDI & PHQ-9 \\
\hline FAS-P & -0.141 & 0.265 & $0.448^{*}$ & $0.673^{* *}$ & 0.356 & $0.456^{*}$ \\
\hline FAS-M & -0.132 & 0.075 & 0.407 & $0.464^{*}$ & $0.658^{* *}$ & $0.749^{* *}$ \\
\hline FAS total & -0.131 & 0.227 & $0.486^{*}$ & $0.693^{* *}$ & $0.607^{* *}$ & $0.669^{* *}$ \\
\hline
\end{tabular}

BDI — Beck Depression Inventory; PHQ-9 — Patient Health Questionnaire; FAS — Fatigue Assessment Scale; FAS-P — Physical Fatigue; FAS-M — Mental Fatigue; ${ }^{*} \mathrm{p}<0.05$ (two-tailed); ${ }^{* *} \mathrm{p}<0.01$ (two-tailed)

und significantly impaired health quality in both post-sarcoidosis fatigue syndrome and active sarcoidosis patients. Depressive symptoms were significantly more frequent in PSFS, comparing to patients with complete remission, but were equally frequent as in active sarcoidosis group. Moreover, health quality was strongly related both to the presence of depressive symptoms as well as to intensity of fatigue.

The Polish version of SHQ has reliability which is acceptable for the purposes of both clinical assessment and scientific research [30], Cronbach's alpha for the SHQ and its dimensions is between 0.78 and 0.95 . Those coefficients are slightly worse than those reported for the original SHQ [18], but comparable to the Japanese adaptation [31]. The validity of the Polish version of the SHQ was verified by means of intergroup comparisons and calculation of correlation quotients. It may be assumed that the adaptation is valid based on the following findings:

1. There was a negative correlation between SHQ scores and the depressive symptoms severity, i.e. the BDI and PHQ-9 scores. Similar relationships between quality of life and depression has been previously reported for different diseases [32-34]. Depression and quality of life are both related to general well-being of the patient and emphasise the biopsychosocial aspects of the chronic disease. The association between them is probably two-way. Those terms may be considered as opposites since usually higher degree of depression is associated with low quality of life.

2. There is a negative correlation between the SHQ scores and FAS scores.

The SHQ scores were higher in PSFS and $S$-A groups than in the S-R group. No association between lung capacity indicators and SHQ score were found. Statistically significant correlations between FVC and $\mathrm{FEV}_{1}$ and SHQ score were reported in the original validation study [18] and only between FVC and SHQ in Japanese adaptation [31]. However, it should be noted that the correlation coefficients were low, which suggests a small effect size. Thus, this results might be replicated in a sample larger than hereby research. The low number of patients with severe sarcoidosis, with severely impaired lung function may be also responsible for the lack of such correlation.

In our work we focused mainly on post-sarcoidosis fatigue syndrome, and the study was not aimed to solve the problem of fatigue etiopathogenesis. We can only resume, that fatigue in general influences the health quality and is related to depressive symptoms. Health quality was equally impaired in PSFS and active sarcoidosis. There was only a non-significant trend towards higher scores in DPI and PHQ-9 questionnaires in PSFS comparing to active sarcoidosis, suggesting the influence of active chronic disease on these symptoms. FAS was positively correlated with depressive symptoms, and this correlation was especially strong in a subgroup analysis of PSFS patients.

We found only weak negative correlation between FAS mental fatigue score and $\mathrm{FEV}_{1}$. Baydur et al found a negative correlation between vital capacity (VC) and mental fatigue assessed by another fatigue questionnaire, a 20-item multidimentional fatigue inventory (MFI-20) [35]. Another group of Polish authors have not found correlations between fatigue severity assessed by FAS and lung function test parameters (spirometry and DLCO) [36]. As already mentioned above, our study groups were not represented by patients with the most severe disease, therefore it is difficult to conclude on the importance of impaired lung function on fatigue based on our results. In our opinion, in the multidimensional 
aspect of fatigue etiopathogenesis impaired lung function should still be considered as a potential cause of fatigue.

In previous studies FAS was proved an excellent unidimensional tool for the assessment of multidimensional syndrome $[8,17]$. Although fatigue in sarcoidosis should be considered as a syndrome of many possible causes and complicated background (multidimensionality) [10], FAS measures fatigue independently of depressive symptoms (it is unidimensional) [17]. Nevertheless, both anxiety and depressive symptoms were shown to be significant predictors of high fatigue scores [16]. In our study we confirmed this links, also in this particular situation, when fatigue persists in patients with radiological and clinical remission.

De Kleijn et al. [37], based on the anchor-based and distribution-based methods, estimated a 4-point difference in the FAS scores to be the minimal clinically important differences (MCID). Although the purpose of the cited study was to find the clinically important difference for longitudinal assessments, it is of note, that in our study mean FAS values between S-R and S-A/PSFS groups were much above this threshold. Also the difference between S-A and PSFS, although not clinically significant, was higher in PSFS group and equalled the MCID value.

In summary, Polish version of SHQ is a valid instrument for the assessment of health quality. Health quality is impaired in post-sarcoidosis fatigue syndrome, when compared to patients with complete remission, and the level of this impairment is comparable to that observed in active sarcoidosis. Health quality is strongly related to depressive symptoms and fatigue. The intensity of depressive symptoms is higher in PSFS comparing to complete remission, but comparable to active disease.

\section{Conflict of interest}

The authors declare no conflict of interest.

\section{References:}

1. Baughman RP, Culver DA, Judson MA. A concise review of pulmonary sarcoidosis. Am J Respir Crit Care Med. 2011; 183(5): 573-581, doi: 10.1164/rccm.201006-0865CI, indexed in Pubmed: 21037016.

2. Iannuzzi MC, Rybicki BA, Teirstein AS. Sarcoidosis. N Engl J Med. 2007; 357(21): 2153-2165, doi: 10.1056/NEJMra071714, indexed in Pubmed: 18032765.

3. Wirnsberger RM, de Vries J, Wouters EF, et al. Clinical presentation of sarcoidosis in The Netherlands an epidemiological study. Neth J Med. 1998; 53(2): 53-60, indexed in Pubmed: 9766153.
4. Michielsen HJ, Drent M, Peros-Golubicic T, et al. Fatigue is associated with quality of life in sarcoidosis patients. Chest. 2006; 130(4): 989-994, doi: 10.1378/chest.130.4.989, indexed in Pubmed: 17035429.

5. Lower EE, Harman S, Baughman RP. Double-blind, randomized trial of dexmethylphenidate hydrochloride for the treatment of sarcoidosis-associated fatigue. Chest. 2008; 133(5): 1189-1195, doi: 10.1378/chest.07-2952, indexed in Pubmed: 18263672.

6. Lower EE, Malhotra A, Surdulescu V, et al. Armodafinil for sarcoidosis-associated fatigue: a double-blind, placebo-controlled, crossover trial. J Pain Symptom Manage. 2013; 45(2): 159-169, doi: 10.1016/j.jpainsymman.2012.02.016, indexed in Pubmed: 22917711.

7. Centers for Disease Control and Prevention. http://www.cdc. gov/cfs/case-definition (28.01.2016).

8. Whitehead L. The measurement of fatigue in chronic illness: a systematic review of unidimensional and multidimensional fatigue measures. J Pain Symptom Manage. 2009; 37(1): 107-128, doi: 10.1016/j.jpainsymman.2007.08.019, indexed in Pubmed: 19111779.

9. Sharma OmP. Fatigue and sarcoidosis. Eur Respir J . 1999; 13: 713-714.

10. Górski W, Piotrowski WJ. Fatigue syndrome in sarcoidosis. Pneumonol Alergol Pol. 2016; 84(4): 244-250, doi: 10.5603/ PiAP.2016.0030, indexed in Pubmed: 27435351.

11. Korenromp IHE, Heijnen CJ, Vogels OJM, et al. Characterization of chronic fatigue in patients with sarcoidosis in clinical remission. Chest. 2011; 140(2): 441-447, doi: 10.1378/ chest.10-2629, indexed in Pubmed: 21330380.

12. Korenromp IHE, Grutters JC, van den Bosch JMM, et al. Post -inflammatory fatigue in sarcoidosis: personality profiles, psychological symptoms and stress hormones. J Psychosom Res. 2012; 72(2): 97-102, doi: 10.1016/j.jpsychores.2011.10.001, indexed in Pubmed: 22281449.

13. Chang B, Steimel J, Moller DR, et al. Depression in sarcoidosis. Am J Respir Crit Care Med. 2001; 163(2): 329-334, doi: 10.1164/ajrccm.163.2.2004177, indexed in Pubmed: 11179101.

14. Holas P, Krejtz I, Urbankowski T, et al. Anxiety, its relation to symptoms severity and anxiety sensitivity in sarcoidosis. Sarcoidosis Vasc Diffuse Lung Dis. 2013; 30(4): 282-288, indexed in Pubmed: 24351619.

15. Goracci A, Fagiolini A, Martinucci M, et al. Quality of life, anxiety and depression in sarcoidosis. Gen Hosp Psychiatry. 2008; 30(5): 441-445, doi: 10.1016/j.genhosppsych.2008.04.010, indexed in Pubmed: 18774427.

16. de Kleijn WPE, Drent M, De Vries J. Nature of fatigue moderates depressive symptoms and anxiety in sarcoidosis. Br J Health Psychol. 2013; 18(2): 439-452, doi: 10.1111/j. 2044-8287.2012.02094.x, indexed in Pubmed: 22988824.

17. De Vries J, Michielsen H, Van Heck GL, et al. Measuring fatigue in sarcoidosis: the Fatigue Assessment Scale (FAS). Br J Health Psychol. 2004; 9(Pt 3): 279-291, doi: 10.1348/1359107041557048, indexed in Pubmed: 15296678.

18. Cox CE, Donohue JF, Brown CD, et al. The Sarcoidosis Health Questionnaire: a new measure of health-related quality of life. Am J Respir Crit Care Med. 2003; 168(3): 323-329, doi: 10.1164/rccm.200211-1343OC, indexed in Pubmed: 12738606.

19. American Thoracic Society, European Respiratory Society, World Association of Sarcoidosis and Other Granulomatous Disorders: Statement on sarcoidosis. Am J Respir Crit Care Med. 1999; 160(2): 736-755, doi: 10.1164/ajrccm.160.2.ats4-99, indexed in Pubmed: 10430755.

20. Polish Society of Respiratory Diseases. The recommendations of the Polish Society of Respiratory Diseases on spirometry. Pneumonol Alergol Pol. 2006; 74(Suppl 1).

21. Sterk PJ, Fabbri LM, Quanjer PH, et al. Standardized lung function testing. Official statement of the European Respiratory Society. Eur Respir J Suppl. 1993; 16: 1-100, indexed in Pubmed: 8499052.

22. Anastasi A, Urbina S. Psychological testing. Prentice 1997.

23. Beck AT, Ward CH, Mendelson M, et al. An inventory for measuring depression. Arch Gen Psychiatry. 1961; 4: 561-571, indexed in Pubmed: 13688369. 
24. Beck A, Steer RA, Brown G. Beck Depression Inventory-II. PsycTESTS Dataset. 2011, doi: 10.1037/t00742-000.

25. Zawadzki B, Popiel A, Pragłowska E. Psychometric properties of the Polish version of the Aaron T. Beck's Depression Inventory BDI. Psychologia — Etologia - Genetyka. 2009; 19: 71-95.

26. Kroenke K, Spitzer RL, Williams JB. The PHQ-9: validity of a brief depression severity measure. J Gen Intern Med. 2001; 16(9): 606-613, indexed in Pubmed: 11556941.

27. http://www.phqscreeners.com/sites/g/files/g10016261/f/201412/ PHQ9 Polish\%20for\%20Poland.pdf. (12.10.2017).

28. WASOG World Association for Sarcoidosis and Other Granulomatous Disorders. http://www.wasog.org/education-research/ questionnaires.html (12.10.2017).

29. http://vassarstats.net/rdiff.html (12.0.2017).

30. Tavakol M, Dennick R. Making sense of Cronbach's alpha. Int J Med Educ. 2011; 2: 53-55, doi: 10.5116/ijme.4dfb.8dfd, indexed in Pubmed: 28029643.

31. Tanizawa K, Handa T, Nagai S, et al. Validation of the Japanese version of the Sarcoidosis Health Questionnaire: a cross-sectional study. Health Qual Life Outcomes. 2011; 9: 34, doi: 10.1186/1477-7525-9-34, indexed in Pubmed: 21569587.

32. Lomper K, Chudiak A, Uchmanowicz I, et al. Effects of depression and anxiety on asthma-related quality of life. Pneumonol Alergol Pol. 2016; 84(4): 212-221, doi: 10.5603/ PiAP.2016.0026, indexed in Pubmed: 27435347.
33. Nguyen C, Ranque B, Baubet T, et al. Groupe Français de Recherche sur la Sclérodermie. Clinical, functional and health-related quality of life correlates of clinically significant symptoms of anxiety and depression in patients with systemic sclerosis: a cross-sectional survey. PLoS One. 2014; 9(2): e90484, doi: 10.1371/journal.pone.0090484, indexed in Pubmed: 24587375.

34. Sivertsen H, Bjørkløf GH, Engedal K, et al. Depression and Quality of Life in Older Persons: A Review. Dement Geriatr Cogn Disord. 2015; 40(5-6): 311-339, doi: 10.1159/000437299, indexed in Pubmed: 26360014.

35. Baydur A, Alavy B, Nawathe A, et al. Fatigue and plasma cytokine concentrations at rest and during exercise in patients with sarcoidosis. Clin Respir J. 2011; 5(3): 156-164, doi: 10.1111/j. 1752-699X.2010.00214.x, indexed in Pubmed: 21679351.

36. Zieleźnik K, Jastrzębski D, Ziora D. Fatigue in patients with inactive sarcoidosis does not correlate with lung ventilation ability or walking distance. Pilot Study. Pneumonol Alergol Pol. 2015; 83(1): 14-22, doi: 10.5603/PiAP.2015.0002, indexed in Pubmed: 25577529.

37. de Kleijn WPE, De Vries J, Wijnen PA, et al. Minimal (clinically) important differences for the Fatigue Assessment Scale in sarcoidosis. Respir Med. 2011; 105(9): 1388-1395, doi: 10.1016/j.rmed.2011.05.004, indexed in Pubmed: 21700440 . 